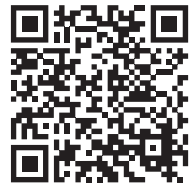

Octubre - Diciembre 2021 Vol. 1, núm. 1 / pp. 7-13

Palabras clave:

Hueso hioides, ángulo mandibular, mentón, clase facial,

rejuvenecimiento facial.

Keywords: Hyoid bone, mandibular angle, chin, facial class, facial rejuvenation.

Citar como: Ravelo V, Haidar ZS,

Huentequeo-Molina

C, Martínez F, Olate S. Posición esqueletal aplicada a la estética y rejuvenecimiento del tercio inferior facial y cervical. Lat Am J Oral Maxillofac Surg. 2021; 1 (1): 7-13. https://dx.doi. org/10.35366/101911

* Centro de Excelencia en Estudios Morfológicos y Quirúrgicos, Universidad de La Frontera, Temuco, Chile. ${ }^{\ddagger}$ BioMAT'X, Facultad de Odontología. Centro de Investigación e Innovación Biomédica (CIIB), Facultad

de Medicina. Universidad de los Andes. Santiago, Chile.

$\S$ Departamento de Cirugía

Oral y Maxilofacial, Hospital «Dr. Abraham Godoy Peña». Lautaro, Chile.

"Departamento de Cirugía Oral y Maxilofacial, Hospital «Dr. Hernán Henríquez Aravena». Temuco, Chile. División de Cirugía Oral, Facial y Maxilofacial, Universidad de La Frontera. Temuco, Chile.

Recibido: 10/08/2021 Aceptado: 17/08/2021

doi: 10.35366/101911

\section{Posición esqueletal aplicada a la estética y rejuvenecimiento del tercio inferior facial y cervical}

\author{
Skeletal position applied to the aesthetic and rejuvenation \\ of the lower facial third and cervical area
}

\section{RESUMEN}

El objetivo de este estudio fue evaluar la posición del hueso hioides y su relación con el plano mandibular y relacionar aspectos para uso en diagnóstico y planificación de técnicas quirúrgicas y no quirúrgicas. Se diseñó un estudio de corte transversal en 115 sujetos que presentaran una tomografía computarizada de haz cónico como elemento diagnóstico. Se determinó la posición sagital y angulación del plano mandibular junto a la posición vertical y sagital del hueso hioides en relación con el plano mandibular. Se utilizó el análisis de concordancia; en las variables categóricas se obtuvo un índice kappa de 0.90 y para las variables continuas se obtuvo un índice intraclase de 0.87; además se utilizó el test de Spearman, considerando un valor $p<0.005$ para determinar la significancia estadística. 71 sujetos con clase II esqueletal presentaron mayor dimensión en $\mathrm{H}^{\mathrm{P}}$-Go $(p=0.0001)$ y 44 sujetos con clase III esqueletal mostraron mayor dimensión $\mathrm{H}^{\mathrm{A}}-\mathrm{Me}$ y $\mathrm{H}^{\mathrm{A}}$-Pog. Se observó la posición retrognática mandibular asociada a mayor distancia $\mathrm{H}^{\mathrm{P}}$-Go $(\mathrm{p}=0.0001)$; hubo una correlación entre la angulación del plano mandibular y las mediciones $\mathrm{H}^{\mathrm{P}}$-Go $(p=0.031)$ y H $\mathrm{H}^{\mathrm{A}}-\mathrm{Me}(\mathrm{p}=0.032)$. Se puede concluir que la posición del hueso hioides se relaciona con los patrones esqueletales. La mandíbula retraída presenta mayor distancia vertical entre el hueso hioides y el ángulo mandibular y debe integrarse en procesos de diagnóstico para la selección de técnicas de cambio de imagen facial y rejuvenecimiento.

\section{ABSTRACT}

The aim of this research was to evaluate the position of the hyoid bone and its relationship with the mandibular plane and to relate aspects for the use in diagnosis and planning of surgical and non-surgical techniques. A cross-sectional study was designed in 115 subjects who presented a cone beam computed tomography as a diagnostic technique. The sagittal position and angle of the mandibular plane were obtained together with the vertical and sagittal position of the hyoid bone in relation to the mandibular plane. Concordance analysis was used; for categorical variables, a kappa index of 0.90 was obtained and for continuous variables, an intraclass index of 0.87 was obtained; in addition, the Spearman test was used, considering a $p$ value $<0.005$ to determine statistical significance. 71 subjects with skeletal class II presented greater dimension in $H^{P}$-Go $(p=0.0001)$ and 44 subjects with skeletal class III presented greater dimension $\mathrm{H}^{\mathrm{A}}$-Me and $\mathrm{H}^{\mathrm{A}}$-Pog. Mandibular retrognathic position associated with greater $H^{P}$-Go distance $(p=$ 0.0001 ) was observed; there was a correlation between mandibular plane angulation and $H^{P}-\mathrm{Go}(p=0.031)$ and $H^{A}$-Me $(p=0.032)$ measurements. It can be concluded that the position of the hyoid bone is related to skeletal patterns. The retruded mandible has a greater vertical distance between the hyoid bone and the mandibular angle and should be integrated in diagnostic processes for the selection of facial makeover and rejuvenation techniques.

\section{INTRODUCCIÓN}

El rostro humano está compuesto por estructuras blandas y duras y puede verse afectado por cambios en el plano profundo o por alteraciones en planos superficiales. ${ }^{1}$ Cambios en la morfología del esqueleto facial implican modificaciones en el tono y posición muscular, modificaciones en las proporciones y cambios en la posición de las estructuras faciales. ${ }^{2,3}$
La posición mandibular, angulación mandibular y posición del hueso hioides tienen estrecha relación y su modificación genera cambios de estética y función. ${ }^{4,5}$ Estos patrones morfológicos se consideran factores de riesgo de desarrollar, entre otras situaciones, el síndrome de apnea o hipoapnea obstructiva del sueño (SAHOS) de gran impacto en la morfología funcional. ${ }^{6,7}$ Por otra parte, en el envejecimiento facial se ha observado una intensa remodelación ósea de la sínfisis y 
parasínfisis mandibular, llevando a un patrón facial con rotación mandibular de tipo horario y características de clase II. ${ }^{8}$

En el análisis estético del tercio inferior, la transición entre el contorno mandibular y el área cervical son importantes para determinar un rostro estético y joven. Esta área está relacionada con: 1) tejidos blandos externos como músculo masetero y tejido adiposo supraplatisma; 2) tejidos blandos internos como glándula submandibular, músculo milohioideo y otros; y 3) posición de ángulo mandibular y hueso hioides. El ángulo mandibular, mentón y hueso hioides se relacionan directamente y definen la posición muscular de la zona. Esta relación desempeña un rol importante en técnicas de rejuvenecimiento, cambio de imagen y en condiciones funcionales del área facial. ${ }^{9}$

En sujetos con deformidades faciales existen cambios en la posición mandibular, maxilar, nasal y del hueso hioides, de forma que el patrón esqueletal puede relacionarse con el patrón facial muscular. ${ }^{2,10}$ El objetivo de este estudio es evaluar la posición del hueso hioides en sentido vertical y sagital y su relación con el plano mandibular en pacientes con deformidad facial, y determinar algunas opciones para su integración en las decisiones de técnicas quirúrgicas para cambio de forma y rejuvenecimiento facial.

\section{Material y métodos}

Se realizó un estudio de corte transversal para identificar la posición sagital y vertical del hueso hioides y su relación

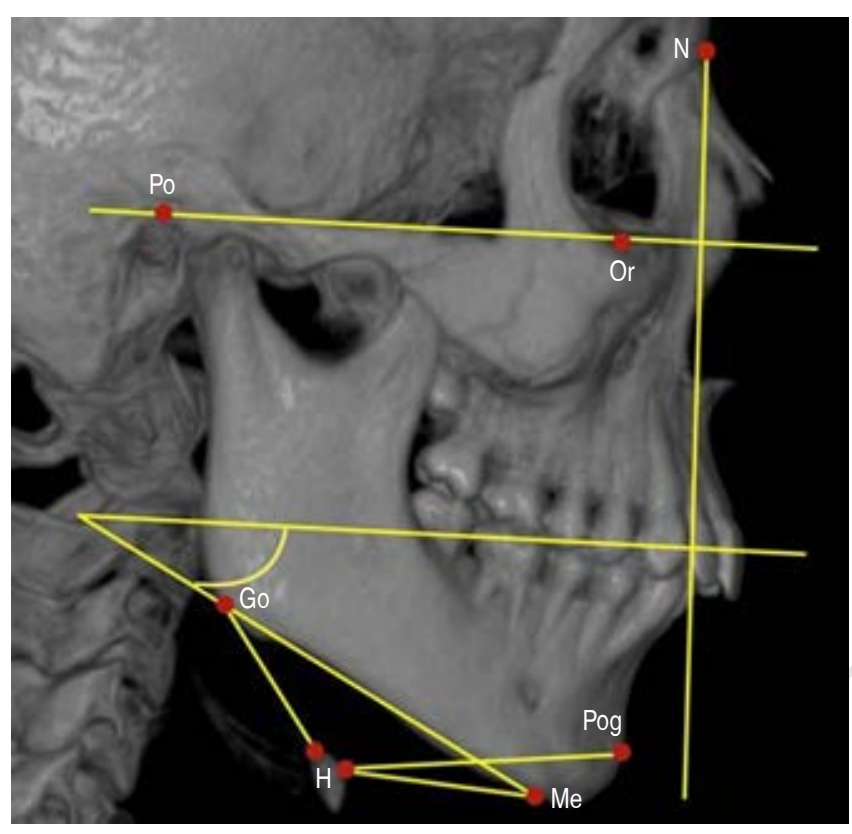

Figura 1: Puntos, líneas y ángulos utilizados en la morfometría facial de sujetos clase II esqueletal.

$N=$ nasión. Po $=$ porion. Or $=$ orbital. $\mathrm{Co}=$ gonion. $\operatorname{Pog}=$ pogonion. $H=$ hioides. Me = mentón

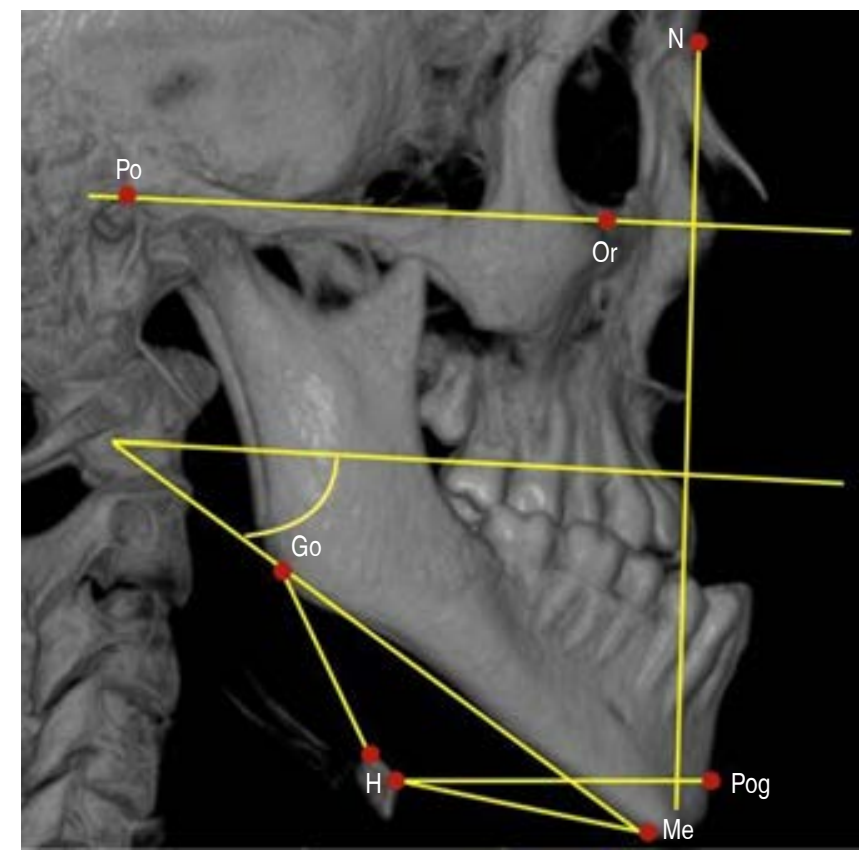

Figura 2: Puntos, líneas y ángulos utilizados en la morfometría facial de sujetos clase III esqueletal.

$N=$ nasión.. o $=$ porion. Or $=$ orbital. $G o=$ gonion.. og $=$ pogonion. $H=$ hioides. Me $=$ mentón .

con la posición sagital de mandíbula y la angulación del plano mandibular. Las imágenes fueron utilizadas para el diagnóstico facial prequirúrgico. Los sujetos incorporados firmaron un consentimiento informado y accedieron voluntariamente al estudio. La investigación se realizó resguardando la integridad de los pacientes y respetando la declaración de Helsinki.

Se incluyeron sujetos mayores de 18 años de ambos sexos que presentaran una deformidad facial clase II (análisis de Steiner con ángulo $>2^{\circ} \pm 2$ ) o que tuvieran una deformidad facial clase III (análisis de Steiner con ángulo $<2^{\circ} \pm 2$ ). Se excluyeron sujetos con cirugía facial previa, antecedentes de trauma facial, presencia de síndromes o condiciones faciales de significativa alteración morfológica y sujetos con asimetrías faciales definidas por la desviación del mentón por más de 5 $\mathrm{mm}$ desde la línea media facial.

Se realizó una captura de imagen con el tomógrafo 3D NewTom, modelo VGi EVO (Verona, Italia), campo de visualización de $24 \times 19 \mathrm{~cm}$ y parámetros de exposición 110 kV, $8 \mathrm{~mA}, 15 \mathrm{~s}$. La imagen fue obtenida por especialistas; el paciente se ubicó en posición vertical, manteniendo los labios en reposo, sin forzar ninguna posición corporal.

Se utilizó el análisis de McNamara para determinar la posición anteroposterior y angulación del plano mandibular en pacientes clase II (Figura 1) y clase III esqueletal (Figura 2). 
Para determinar la posición vertical y sagital del hueso hioides se utilizó la metodología propuesta anteriormente. ${ }^{11}$

\section{Posición mandibular}

Se utilizó el plano de Frankfurt formado por los puntos Po-Or (porión: punto más superior del conducto auditivo externo; orbitario: punto más inferior de la órbita) y se trazó una línea perpendicular que pasa por el punto $\mathrm{N}$ (nasión: punto más anterior de la sutura frontonasal). Para determinar la posición anteroposterior de la mandíbula se midió la distancia del punto Pog (pogonión: punto más anterior de la sínfisis mandibular) a la línea perpendicular desde nasión (parámetro de normalidad $2 \pm 4 \mathrm{~mm})$.

\section{Angulación del plano mandibular}

Se midió la angulación de la intersección de los puntos Po-Or y Go-Me (gonión: punto localizado en la parte más posterior e inferior del ángulo mandibular; Me: punto más inferior de la sínfisis mandibular) con parámetro de normalidad de $25^{\circ} \pm 4^{\circ}$.

\section{Posición hueso hioides}

Sagital: se midió la distancia entre los puntos $\mathrm{H}^{\mathrm{A}}-\mathrm{Me}$ (hioides ${ }^{\mathrm{A}}$ : punto anterior más superior del cuerpo del hueso hioides) y los puntos $\mathrm{H}^{\mathrm{A}}$-Pog.

Vertical: se midió la distancia entre los puntos $\mathrm{H}^{\mathrm{P}}-\mathrm{Go}$ (hioides ${ }^{\mathrm{P}}$ : punto posterior más superior del cuerpo del hueso hioides).

Las mediciones fueron realizadas por el mismo observador en distintos tiempos clínicos. Se utilizó el análisis de concordancia en 30 estudios con un intervalo de tres semanas. Para las variables categóricas se obtuvo un índice kappa $=$ 0.90 y para las variables continuas se obtuvo un índice intraclase de 0.87 . Se utilizó un intervalo de confianza de 95\%

Tabla 1: Distribución de los sujetos incluidos en relación con la posición del hueso hioides en sentido sagital y vertical.

\begin{tabular}{|c|c|c|c|}
\hline \multirow{2}{*}{$\begin{array}{l}\text { Posición del } \\
\text { hueso hioides }\end{array}$} & \multicolumn{2}{|c|}{ Media $\pm \mathrm{DE}(\mathrm{mm})$} & \multirow[b]{2}{*}{$p<0.05$} \\
\hline & Masculino & Femenino & \\
\hline$H^{P}-G_{0}$ & $28.84 \pm 5.32$ & $26.36 \pm 4.27$ & $0.006^{*}$ \\
\hline $\mathrm{H}^{\mathrm{A}}-\mathrm{Me}$ & $34.41 \pm 5.52$ & $34.04 \pm 4.84$ & 0.40 \\
\hline$H^{A}-P o g$ & $43.84 \pm 6.10$ & $42.39 \pm 4.83$ & $0.034^{*}$ \\
\hline
\end{tabular}

$H^{P}=$ porción posterior del hueso hioides; $H^{A}=$ porción anterior del hueso hioides; Go = gonión; $\mathrm{Me}$ = mentón; $\mathrm{Pog}$ = pogonión; $\mathrm{DE}$ = desviación estándar.

* Indica diferencia estadística significativa.

Tabla 2: Distribución de los sujetos incluidos en
relación con la clase esqueletal y la posición del
hueso hioides en sentido sagital y vertical.

para medir la concordancia de la posición del hueso hioides, la posición mandibular y angulación del plano mandibular. Además, se utilizó el test de Spearman para determinar la correlación entre las variables y su relación con la clase esqueletal. Se consideró un valor $\mathrm{p}<0.05$ para determinar la significancia estadística.

\section{Resultados}

Se incluyeron 115 sujetos, $40 \%$ del sexo masculino y $60 \%$ del sexo femenino con una edad promedio de 25 años. Al analizar la posición del hueso hioides y el sexo de los sujetos estudiados (Tabla 1) se observó que el sexo masculino mostró mayor distancia en $\mathrm{H}^{\mathrm{P}}-\mathrm{Go}(\mathrm{p}=0.006)$ y $\mathrm{H}^{\mathrm{A}}-\mathrm{Pog}(\mathrm{p}=0.034)$ que el sexo femenino.

Según la clasificación propuesta por Steiner, 71 sujetos presentaron una clase II esqueletal, quienes mostraron una distancia significativamente mayor en $\mathrm{H}^{\mathrm{P}}$-Go $(\mathrm{p}=0.0001)$. Por otra parte, 44 sujetos presentaron clase III esqueletal, y mostraron mayor distancia $\mathrm{H}^{\mathrm{A}}-\mathrm{Me}$ y $\mathrm{H}^{\mathrm{A}}-\mathrm{Pog}(\mathrm{p}=0.0001)$ (Tablas 2 y 3 ).

Con respecto a la angulación del plano mandibular hubo una correlación significativa entre ésta y la medición $\mathrm{H}^{\mathrm{P}}$-Go $(\mathrm{p}$ $=0.031$ ), indicando que, al aumentar el ángulo mandibular, existía mayor distancia vertical entre hueso hioides y gonión $(\mathrm{H}-\mathrm{Go})$. De la misma forma, a mayor distancia sagital de las mediciones $\mathrm{H}^{\mathrm{A}}-\mathrm{Me}(\mathrm{p}=0.032)$ y $\mathrm{H}^{\mathrm{A}}$-Pog $(\mathrm{p}=0.13)$ se observó menor ángulo mandibular (Tabla 4).

\section{Discusión}

Las imágenes 2D y 3D son útiles para realizar análisis de diagnóstico y morfología facial; sin embargo, la imagen 3D nos permite llevar a cabo estudios profundos y detallados de 
las condiciones faciales a nivel de tejidos blandos y tejidos duros. ${ }^{12,13}$

Se ha indicado ${ }^{14}$ que la posición del hueso hioides no es constante, y que depende de las relaciones anteroposteriores maxilomandibulares. En la presente investigación se observó una correlación significativa entre la posición anteroposterior de la mandíbula, el ángulo mandibular y la posición del hueso hioides; en los sujetos clase II facial se relacionó la posición retrognática mandibular con el aumento en el ángulo del plano mandibular y aumento en la distancia del ángulo mandibular al hueso hioides en comparación con lo observado en los sujetos clase III facial.

En 182 sujetos se demostró ${ }^{5}$ una correlación positiva entre patrón esqueletal y posición del hueso hioides; sujetos con características como una rama mandibular de menor tamaño, un perfil convexo y rotación horaria de la mandíbula presentaron un hueso hioides orientado hacia posterior y en posición más inferior. Con estos resultados es posible definir un patrón esqueletal y consecuentemente un patrón muscular y de posición de paquetes de grasa facial inferior y cervical en relación con el hueso hioides y contorno mandibular.

Estos aspectos son interesantes de observar debido al impacto del envejecimiento en el esqueleto facial y la remodelación ósea que se han descrito intensamente en los últimos 15 años, ${ }^{15}$ considerando que muchos pacientes sometidos a técnicas de rejuvenecimiento requieren de intervenciones estéticas como el levantamiento facial (lifting) o la plicatura de músculo platisma en la región submandibular. ${ }^{16}$

En este sentido, se ha definido que el envejecimiento se relaciona con un aumento del ancho orbitario, aumento transversal de la abertura piriforme, aumento del ángulo maxilar y reducción del mentón y contorno lateral de la mandíbula, ${ }^{17}$ mientras que otros autores indicaron ${ }^{18}$ que el

Tabla 3: Distribución de los sujetos incluidos en relación con la posición anteroposterior de la mandíbula y la posición del hueso hioides en sentido sagital y vertical.

\begin{tabular}{cccc} 
& \multicolumn{3}{c}{$\begin{array}{c}\text { Posición sagital de mandíbula } \\
\text { Media } \pm \mathrm{DE}(\mathrm{mm})\end{array}$} \\
\cline { 2 - 3 } $\begin{array}{c}\text { Posición del } \\
\text { hueso hioides }\end{array}$ & Retrognática & Prognática & $\mathrm{p}<0.05$ \\
\hline $\mathrm{H}^{\mathrm{P}}-\mathrm{Go}$ & $29.82 \pm 4.50$ & $25.21 \pm 4.25$ & $0.0001^{*}$ \\
$\mathrm{H}^{\mathrm{A}}-\mathrm{Me}$ & $31.48 \pm 4.30$ & $36.41 \pm 4.86$ & $0.0001^{*}$ \\
$\mathrm{H}^{\mathrm{A}}-\mathrm{Pog}$ & $39.97 \pm 4.20$ & $45.38 \pm 5.44$ & $0.0001^{*}$ \\
\hline
\end{tabular}

$H^{P}=$ porción posterior del hueso hioides; $H^{A}=$ porción anterior del hueso hioides; Go = gonión; Me = mentón; Pog = pogonión; DE = desviación estándar.

* Indica diferencia estadística significativa.
Tabla 4: Distribución de los sujetos incluidos en relación con la angulación del plano mandíbula y la posición del hueso hioides en sentido sagital y vertical.

\begin{tabular}{cccc} 
& \multicolumn{2}{c}{$\begin{array}{c}\text { Angulación del plano mandibular } \\
\text { Media } \pm \mathrm{DE}(\mathrm{mm})\end{array}$} \\
\cline { 2 - 3 } $\begin{array}{c}\text { Posición del } \\
\text { hueso hioides }\end{array}$ & $\begin{array}{c}\text { Cerrado / } \\
\text { Convergente }\end{array}$ & $\begin{array}{c}\text { Abierto / } \\
\text { Divergente }\end{array}$ & \multicolumn{1}{c}{$\mathrm{p}<0.05$} \\
\hline $\mathrm{H}^{\mathrm{P}}$-Go & $25.96 \pm 4.91$ & $28.34 \pm 4.35$ & $0.031^{*}$ \\
$\mathrm{H}^{\mathrm{A}}-\mathrm{Me}$ & $36.54 \pm 4.65$ & $32.65 \pm 4.64$ & $0.032^{*}$ \\
$\mathrm{H}^{\mathrm{A}}-\mathrm{Pog}$ & $45.66 \pm 5.38$ & $41.04 \pm 4.90$ & 0.13 \\
\hline
\end{tabular}

$H^{P}=$ porción posterior del hueso hioides; $H^{A}=$ porción anterior del hueso hioides; $\mathrm{Go}$ = gonión; $\mathrm{Me}=$ mentón; $\mathrm{Pog}$ = pogonión; $\mathrm{DE}$ = desviación estándar.

* Indica diferencia estadística significativa.

envejecimiento presenta un patrón esqueletal de rotación horaria asemejando principalmente una condición de clase II esqueletal. Debido a esto, es posible plantear la hipótesis de que la posición de ángulo mandibular, mentón y hueso hioides pueden tener un alto impacto en el envejecimiento facial (Figura 3) y consecuentemente, en la selección de técnicas para su manejo.

La reducción vertical de rama mandibular, asociada a una mayor distancia entre ángulo mandibular y hueso hioides, se puede relacionar con reabsorción condilar u osteoartrosis, ${ }^{19}$ con frecuencia observada en algunos sujetos con clase II esqueletal, lo cual genera deficiencias en el aspecto estético por reducción del contorno facial además de las alteraciones funcionales ampliamente descritas. ${ }^{19}$ De la misma forma, es posible observar la reducción de la distancia entre el mentón y el ángulo cervical, aumentando la posibilidad de ptosis de esta región submandibular, lo cual es característico del envejecimiento facial y con frecuencia asociado a tratamientos con levantamiento facial profundo. ${ }^{20}$

El impacto facial de tejidos blandos generados por la relación entre las estructuras óseas descritas en esta investigación se relaciona con la posición del músculo bucal en la línea oblicua externa de la mandíbula, la posición del músculo milohioideo, el músculo digástrico, la posición de músculos suprahioideos, la posición de la glándula submandibular y la posición de los paquetes de grasa que se observan en la región. ${ }^{16}$ Varios autores ${ }^{21-23}$ concuerdan en que la posición del hueso hioides se adapta y se mueve en conjunto con estructuras anatómicas adyacentes para obtener funciones fisiológicas necesarias para la vida. De esta forma, la posición ósea del tercio inferior de la cara determina la posición muscular, y con ello el patrón facial y probablemente el modelo 
de envejecimiento del paciente. Es necesario determinar el patrón facial en la fase de diagnóstico para considerar las técnicas quirúrgicas y no quirúrgicas que se utilizarán para el cambio de imagen facial o rejuvenecimiento facial.

Se ha propuesto ${ }^{15}$ que el modelo de reabsorción ósea facial del envejecimiento se asocia a: 1) reducción del estrés y requerimiento mecánico posterior a la erupción de los dientes permanentes y 2) permanente movilidad de músculos y ligamentos en áreas estratégicas como algunas zonas orbitarias, nasales y perinasales y de la zona de sínfisis y parasínfisis mandibular. También señalaron que un rostro de contorno y morfología normal en la edad de 20 años tendría mejores opciones de obtener un envejecimiento de menor intensidad al compararlo con sujetos con grados de anormalidad de la morfología facial. ${ }^{15}$

Técnicas sobre tejidos blandos como el levantamiento superficial o profundo se basan fundamentalmente en la reposición de tejidos musculares, ligamentos y paquetes de grasa; de hecho, algunos casos de levantamiento facial profundo incluyen reposición de glándula submandibular o el retiro de la misma. ${ }^{16}$ La estabilidad en estos procesos se ha evaluado positivamente; sin embargo, en la mayoría de las investigaciones revisadas hay un limitado análisis sobre los patrones esqueletales de los sujetos incluidos en las muestras de estudio, de forma que los autores de la presente investigación sugieren la probabilidad de que algunas indicaciones de técnicas de cirugía estética y de rejuvenecimiento facial sobre tejidos blandos se realicen en sujetos con algún patrón esqueletal patológico como la deformidad facial de clase II y que no son evaluados de manera adecuada o incluidos en las muestras de estudios previamente publicados. Las técnicas de osteotomía de avance podrían ser más eficientes y efectivas en estas condiciones, abordando de manera integral la reposición de tejidos blandos duros. ${ }^{24}$
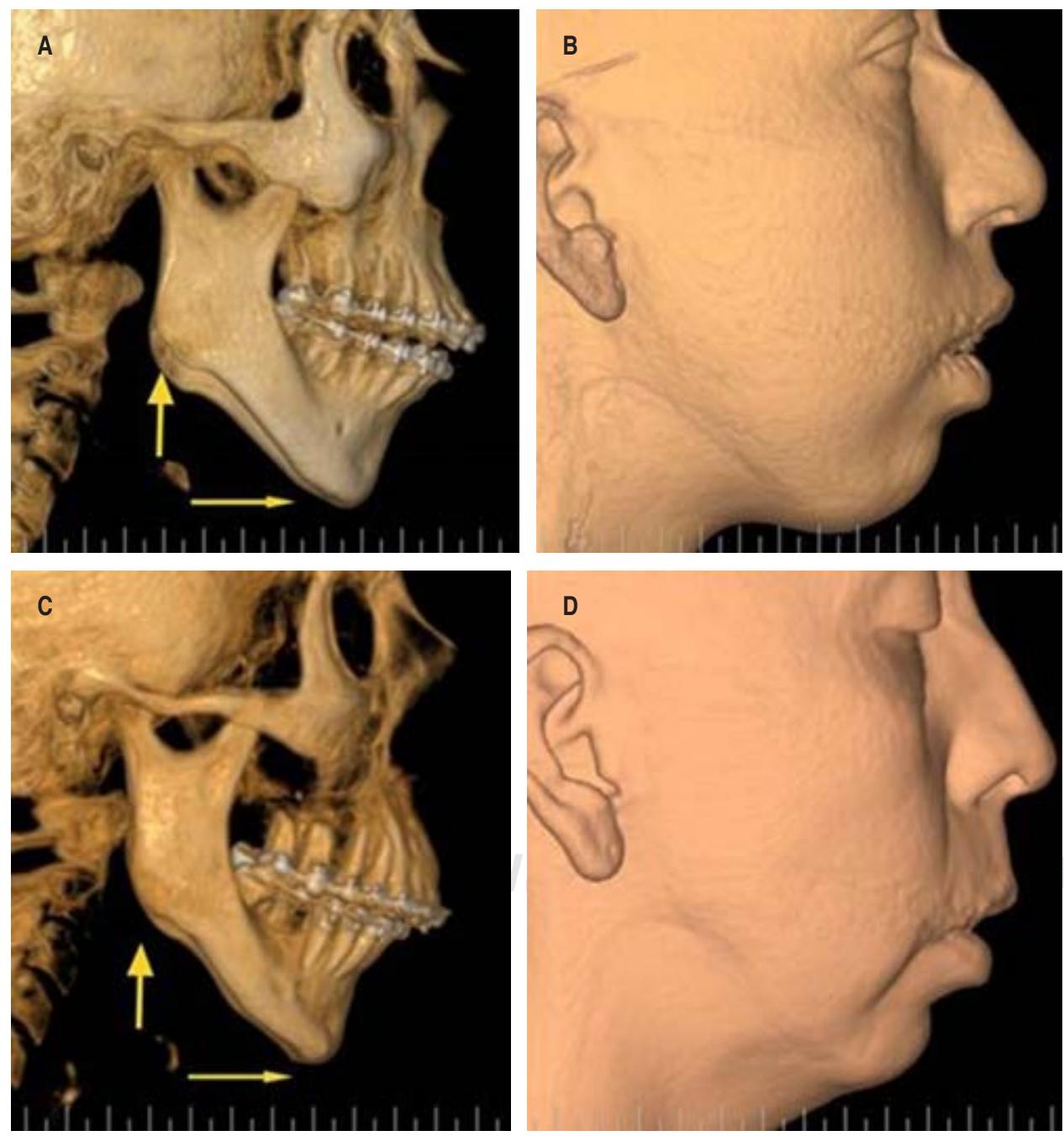

Figura 3:

A y $\boldsymbol{B})$ Paciente de sexo femenino con alteración facial de clase II, con posición retruída de mandíbula, mentón retrasado y rotación facial horaria. C y D) Paciente con características faciales semejantes a la paciente anterior, en edad de 45 años, evidenciando patrones de remodelación ósea con reducción del ángulo mandibular y aumento en rotación facial de sentido horario. 


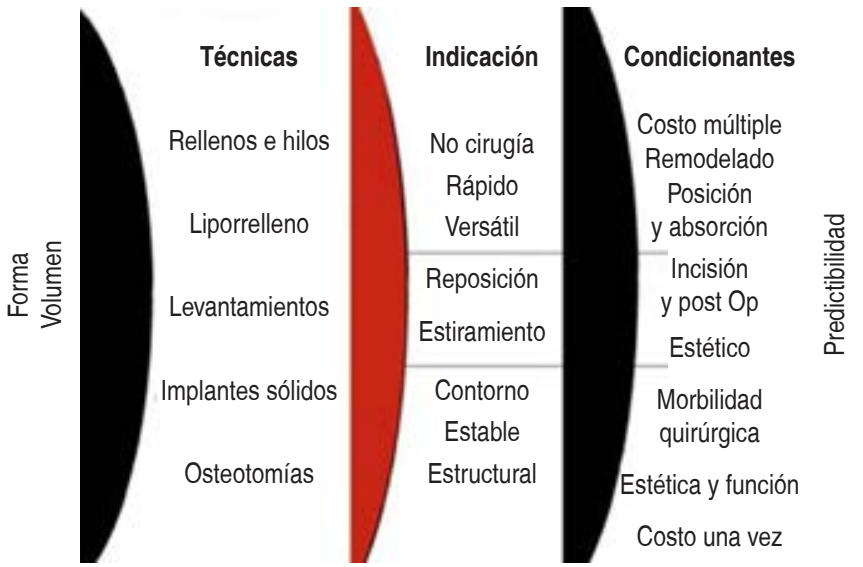

Figura 4: Técnicas utilizadas habitualmente para las modificaciones y rejuvenecimiento facial con manejo de forma y volumen con opciones no quirúrgicas y quirúrgicas.

Procedimientos no quirúrgicos con el uso de hilos y rellenos se han extendido ampliamente en los últimos años, ${ }^{25,26}$ aumentando las herramientas disponibles para los clínicos considerando sus ventajas y desventajas; sin embargo, y de la misma forma que ocurre en las investigaciones de orientación quirúrgica, es necesario definir variables de patrón esqueletal de los sujetos de muestra para definir de mejor forma las indicaciones óptimas de tratamiento. En algún punto todas las técnicas pueden ser complementarias, siendo cada vez más necesario definir las mejores opciones para nuestros pacientes (Figura 4).

Es posible concluir que la posición del ángulo mandibular, mentón y hueso hioides se correlaciona significativamente con el patrón esqueletal. Debe considerarse el patrón esqueletal cuando se seleccionan técnicas para el cambio de imagen o el rejuvenecimiento facial.

\section{ReferenCiAs}

1. Ferrari Júnior FM, Ayub PV, Capelozza Filho L, Pereira Lauris JR, Garib DG. Esthetic evaluation of the facial profile in rehabilitated adults with complete bilateral cleft lip and palate. J Oral Maxillofac Surg. 2015; 73: 169.e1-6.

2. Danahey DG, Dayan SH, Benson AG, Ness JA. Importance of chin evaluation and treatment to optimizing neck rejuvenation surgery. Facial Plast Surg. 2001; 17 (2): 91-97.

3. Sonoda N, Tamatsu Y. Observation on the attachment of muscles onto the hyoid bone in human adults. Okajimas Folia Anat Jpn. 2008; 85 (3): 79-90.

4. Joseph AA, Elbaum J, Cisneros GJ, Eisig SB. A cephalometric comparative study of the soft tissue airway dimensions in persons with hyperdivergent and normodivergent facial patterns. J Oral Maxillofac Surg. 1998; 56 (2): 135-139; discussion 139-140.

5. Jung SK, Kim TW. The relevance analysis of hyoid bone position to skeletal or dental openbite and dentofacial characteristics. Oral Surg Oral Med Oral Pathol Oral Radiol. 2015; 120 (4): 528-533.
6. Neelapu BC, Kharbanda OP, Sardana HK, Balachandran R, Sardana $\mathrm{V}$, Kapoor P, et al. Craniofacial and upper airway morphology in adult obstructive sleep apnea patients: A systematic review and metaanalysis of cephalometric studies. Sleep Med Rev. 2017; 31: 79-90.

7. Ryu HH, Kim CH, Cheon SM, Bae WY, Kim SH, Koo SK, et al. The usefulness of cephalometric measurement as a diagnostic tool for obstructive sleep apnea syndrome: a retrospective study. Oral Surg Oral Med Oral Pathol Oral Radiol. 2015; 119 (1): 20-31.

8. Romo T, Yalamanchili H, Sclafani AP. Chin and prejowl augmentation in the management of the aging jawline. Facial Plast Surg. 2005; 21 (1): 38-46.

9. Yamashita AL, Iwaki Filho L, Leite PCC, Navarro RL, Ramos AL, Previdelli ITS, et al. Three-dimensional analysis of the pharyngeal airway space and hyoid bone position after orthognathic surgery. J Craniomaxillofac Surg. 2017; 45 (9): 1408-1414.

10. Ravelo V, Sigua-Rodriguez EA, Haidar ZS, Brito L, Parra M, Olate S. Impact of facial bone deformity on nasal shape. Plast Aesthet Res. 2021; 8: 19.

11. Mortazavi S, Asghari-Moghaddam H, Dehghani M, Aboutorabzade M, Yaloodbardan B, Tohidi E, et al. Hyoid bone position in different facial skeletal patterns. J Clin Exp Dent. 2018; 10 (4): e346-e351.

12. da Costa ED, Roque-Torres GD, Brasil DM, Bóscolo FN, de Almeida SM, Ambrosano GMB. Correlation between the position of hyoid bone and subregions of the pharyngeal airway space in lateral cephalometry and cone beam computed tomography. Angle Orthod. 2017; 87 (5): 688-695.

13. Kim SH, Choi SK. Changes in the hyoid bone, tongue, and oropharyngeal airway space after mandibular setback surgery evaluated by cone-beam computed tomography. Maxillofac Plast Reconstr Surg. 2020; 42 (1): 27.

14. Deljo E, Filipovic M, Babacic R, Grabus J. Correlation analysis of the hyoid bone position in relation to the cranial base, mandible and cervical part of vertebra with particular reference to bimaxillary relations / teleroentgenogram analysis. Acta Inform Med. 2012; 20 (1): 25-31

15. Mendelson B, Wong $\mathrm{CH}$. Changes in the facial skeleton with aging: implications and clinical applications in facial rejuvenation. Aesthetic Plast Surg. 2012; 36 (4): 753-760.

16. Sykes J, Riedler K, Cotofana S, Palhazi P. Superficial and deep facial anatomy and its implications for rhytidectomy. Facial Plast Surg Clin N Am. 2020; 28: 243-251.

17. Shaw RB Jr, Katzel EB, Koltz PF, Kahn DM, Puzas EJ, Langstein HN. Facial bone density: effects of aging and impact on facial rejuvenation. Aesthet Surg J. 2012; 32 (8): 937-942.

18. Zadoo VP, Pessa JE. Biological arches and changes to the curvilinear form of the aging maxilla. Plast Reconstr Surg. 2000; 106 (2): 460466; discussion 467-468.

19. Bergamaschi IP, Cavalcante RC, Fanderuff M, Gerber JT, Petinati MFP, Sebastiani AM, et al. Orthognathic surgery in class II patients: a longitudinal study on quality of life, TMD, and psychological aspects. Clin Oral Investig. 2021; 25 (6): 3801-3808.

20. Gordon NA, Adam SI 3rd. Deep plane face lifting for midface rejuvenation. Clin Plast Surg. 2015; 42 (1): 129-142.

21. Haralabakis NB, Toutountzakis NM, Yiagtzis SC. The hyoid bone position in adult individuals with open bite and normal occlusion. Eur J Orthod. 1993; 15 (4): 265-271.

22. Hamilton MM, Chan D. Adjunctive procedures to neck rejuvenation. Facial Plast Surg Clin North Am. 2014; 22 (2): 231-242.

23. Jena AK, Duggal R. Hyoid bone position in subjects with different vertical jaw dysplasias. Angle Orthod. 2011; 81 (1): 81-85.

24. Bral A, Olate S, Zaror C, Mensink G, Coscia G, Mommaerts MY. A prospective study of soft- and hard-tissue changes after mandibular 
advancement surgery: midline changes in the chin area. Am J Orthod Dentofacial Orthop. 2020; 157 (5): 662-667.

25. Ali YH. Two years' outcome of thread lifting with absorbable barbed PDO threads: innovative score for objective and subjective assessment. J Cosmet Laser Ther. 2018; 20 (1): 41-49.

26. Hernandez CA, Freytag DL, Gold MH, Pavicic T, Ascher B, de Almeida AT et al. Clinical validation of the temporal lifting technique using soft tissue fillers. J Cosmet Dermatol. 2020; 19 (10): 2529-2535.
Conflicto de intereses: Los autores no reportan conflicto de intereses.

Financiamiento: Los autores declaran no recibir fondos para esta investigación.

\section{Correspondencia:} Prof. Sergio Olate

E-mail: sergio.olate@ufrontera.cl 